Supporting Information

\title{
Single-Molecule Studies Reveal That Water Is a Special Solvent for Amylose and Natural Cellulose
}

Lu Qian, ${ }^{\dagger}$ Wanhao Cai, ${ }^{\dagger}$ Duo Xu, ${ }^{\ddagger}$ Yu Bao,${ }^{\dagger}$ Zhong-yuan Lu* and Shuxun Cui*, ${ }^{\ddagger}$

$\uparrow$ Key Laboratory of Advanced Technologies of Materials (Ministry of Education), Southwest Jiaotong University, Chengdu 610031, China

* State Key Laboratory of Supramolecular Structure and Materials, Institute of Theoretical Chemistry, Jilin University, Changchun 130023, China

E-mail: cuishuxun@swjtu.edu.cn

Tel/Fax: +86-28-87600998 


\section{The Details of the MD Simulations}

The initial conformation of amylose was build according to the data reported by Imberty et al. ${ }^{1}$ The simulation of natural cellulose (NC) was started from the conformation with the angles $\varphi=\angle\left(\mathrm{O} 5, \mathrm{C} 1, \mathrm{O} 4^{\prime}, \mathrm{C}^{\prime}{ }^{\prime}\right)=-120^{\circ}$ and $\psi=\angle$ $\left(\mathrm{C} 1, \mathrm{O} 4^{\prime}, \mathrm{C}^{\prime}, \mathrm{C} 5^{\prime}\right)=-120^{\circ}{ }^{2}$ The simulation system consists of one solute molecule solvated by the simple-point-charge (SPC) water molecules within a cubic simulation box. The box side lengths are $7 \mathrm{~nm}$ for $\mathrm{NC}$ and $4 \mathrm{~nm}$ for amylose, respectively. The systems were simulated under periodic boundary conditions at a temperature of $300 \mathrm{~K}$. The steepest-descent energy minimization was performed first to relax the unfavorable solute-solvent contacts. Electrostatic interactions were treated using the particle-mesh Ewald (PME) method with a real-space cutoff of $1.0 \mathrm{~nm}$. Van der Waals interactions were cut off at $1.0 \mathrm{~nm}$ with the long-range dispersion correction for both energy and pressure. All bond lengths were constrained by the LINCS algorithm. The initial velocities of the atoms were assigned from a Maxwell distribution centered at $300 \mathrm{~K}$. The densities of the systems reached $0.990 \mathrm{~g} / \mathrm{cm}^{3}$ for $\mathrm{NC}$ and $0.978 \mathrm{~g} / \mathrm{cm}^{3}$ for amylose, respectively, after the equilibration run in 100 ps NVT followed by 200 ps NPT ensemble. The production runs of NC and amylose were carried out in an NVT ensemble for $10 \mathrm{~ns}$ with 1 fs integration timestep, respectively. The RMSDs were always around 0.04 for $\mathrm{NC}$ and 0.038 for amylose, which indicated that during the production run the conformations of $\mathrm{NC}$ and amylose did not change much. The number of H-bond between sugar ring and water in 9-10 ns was calculated setting the distance between the $\mathrm{O}$ atom in water and $\mathrm{O}$ atom in $\mathrm{NC}$ or amylose at $0.35 \mathrm{~nm}$ and 
the angle $\mathrm{H}-\mathrm{O}-\mathrm{O}$ at $30^{\circ} .^{3}$

\section{Interfacial Free Energy between Polymer and Solvent}

Recently, Walker and Li found that the plateau height in the $F$-E curves of a hydrophobic polymer (polystyrene) is proportional to the polymer-solvent interfacial free energy: ${ }^{4}$

$$
F_{\text {plateau }} \propto \gamma_{\text {pol-sol }}
$$

where $\gamma_{\text {pol-sol }}$ is the interfacial free energy between polymer and solvent. The interfacial free energy $\gamma_{\text {pol-sol }}$ can be calculated by the Wu's equation:, 6

$$
\gamma_{\mathrm{pol}-\mathrm{sol}}=\gamma_{\mathrm{pol}}+\gamma_{\mathrm{sol}}-\left(\frac{4 \gamma_{\mathrm{pol}}^{\mathrm{d}} \gamma_{\mathrm{sol}}^{\mathrm{d}}}{\gamma_{\mathrm{pol}}^{\mathrm{d}}+\gamma_{\mathrm{sol}}^{\mathrm{d}}}\right)-\left(\frac{4 \gamma_{\mathrm{pol}}^{\mathrm{p}} \gamma_{\mathrm{sol}}^{\mathrm{p}}}{\gamma_{\mathrm{pol}}^{\mathrm{p}}+\gamma_{\mathrm{sol}}^{\mathrm{p}}}\right)
$$

with

$$
\begin{gathered}
\gamma_{\text {pol }}=\gamma_{\text {pol }}^{\mathrm{d}}+\gamma_{\text {pol }}^{\mathrm{p}} \\
\gamma_{\mathrm{sol}}=\gamma_{\mathrm{sol}}^{\mathrm{d}}+\gamma_{\mathrm{sol}}^{\mathrm{p}}
\end{gathered}
$$

where $\gamma_{\mathrm{pol}}$ and $\gamma_{\mathrm{sol}}$ are the surface tensions of polymer and the solvent, $\gamma_{\mathrm{pol}}^{\mathrm{d}}$ and $\gamma_{\text {sol }}^{\mathrm{d}}$ are the dispersive contributions to the surface tensions of polymer and solvent, $\gamma_{\text {pol }}^{\mathrm{p}}$ and $\gamma_{\text {sol }}^{\mathrm{p}}$ are the polar contributions to the surface tensions of polymer and solvent.

The interfacial free energy of $\mathrm{NC}$ in $\mathrm{KCl}$ solutions with different salt concentrations can be calculated by eq S2. As shown in Table S1, the interfacial free energy between $\mathrm{NC}$ and a $\mathrm{KCl}$ solution is increased with the increasing of salt concentration. 


\section{Supporting Data}

Table S1. Dispersive and polar contributions to surface tensions of the solvent used in this study as well as the interfacial free energy between NC and solvent.

\begin{tabular}{|c|c|c|c|c|}
\hline Solvent & $\gamma_{\mathrm{sol}}\left(\mathrm{mJ} / \mathrm{m}^{2}\right)$ & $\gamma_{\mathrm{sol}}^{\mathrm{d}}\left(\mathrm{mJ} / \mathrm{m}^{2}\right)$ & $\gamma_{\mathrm{sol}}^{\mathrm{p}}\left(\mathrm{mJ} / \mathrm{m}^{2}\right)$ & $\gamma_{\mathrm{NC}-\mathrm{sol}}\left(\mathrm{mJ} / \mathrm{m}^{2}\right)$ \\
\hline $1.0 \mathrm{M} \mathrm{KCl}$ in water ${ }^{4,7}$ & 74.2 & 22.1 & 52.1 & 38.0 \\
\hline $0.1 \mathrm{M} \mathrm{KCl}$ in water ${ }^{4,7}$ & 72.8 & 22.1 & 50.7 & 36.8 \\
\hline DI water ${ }^{4}$ & 72.6 & 22.1 & 50.5 & 36.6 \\
\hline Polymer & $\gamma_{\mathrm{NC}}\left(\mathrm{mJ} / \mathrm{m}^{2}\right)$ & $\gamma_{\mathrm{NC}}^{\mathrm{d}}\left(\mathrm{mJ} / \mathrm{m}^{2}\right)$ & $\gamma_{\mathrm{NC}}^{\mathrm{p}}\left(\mathrm{mJ} / \mathrm{m}^{2}\right)$ & \\
\hline $\mathrm{NC}^{8}$ & 66.1 & 50.0 & 16.1 & \\
\hline
\end{tabular}

Table S2. Normalized solvent polarity (NSP) of nonane, DMSO and water. ${ }^{9}$

\begin{tabular}{cccc}
\hline Solvent & nonane & DMSO & water \\
\hline NSP & 0.009 & 0.444 & 1.0 \\
\hline
\end{tabular}




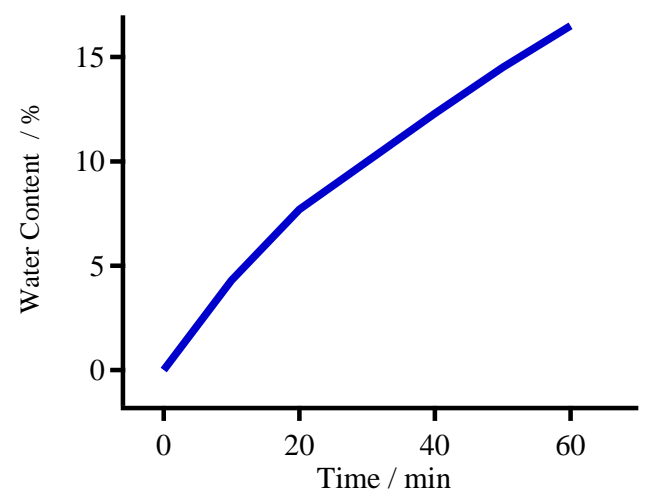

Figure S1. The water absorption speed of DMSO. Experimental details: A substrate is put on an electrical balance (BAS124S, Sartorius). About $0.4 \mathrm{~g}$ of DMSO (similar size to that used in force measurements when the sealed liquid cell is not used) is dropped on the substrate. Then the weight of the droplet is recorded every several minutes. Accordingly, the water content of the droplet vs time can be plotted.
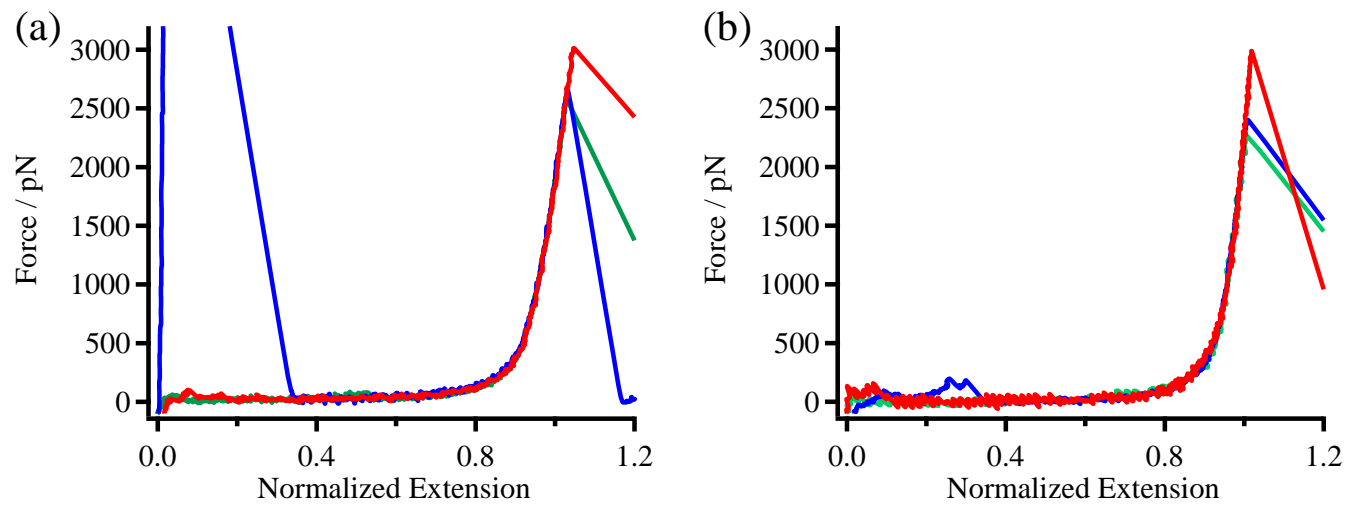

Figure S2. Normalized single-chain $F$ - $E$ curves of NC obtained in nonane (a) and DMSO (b). 

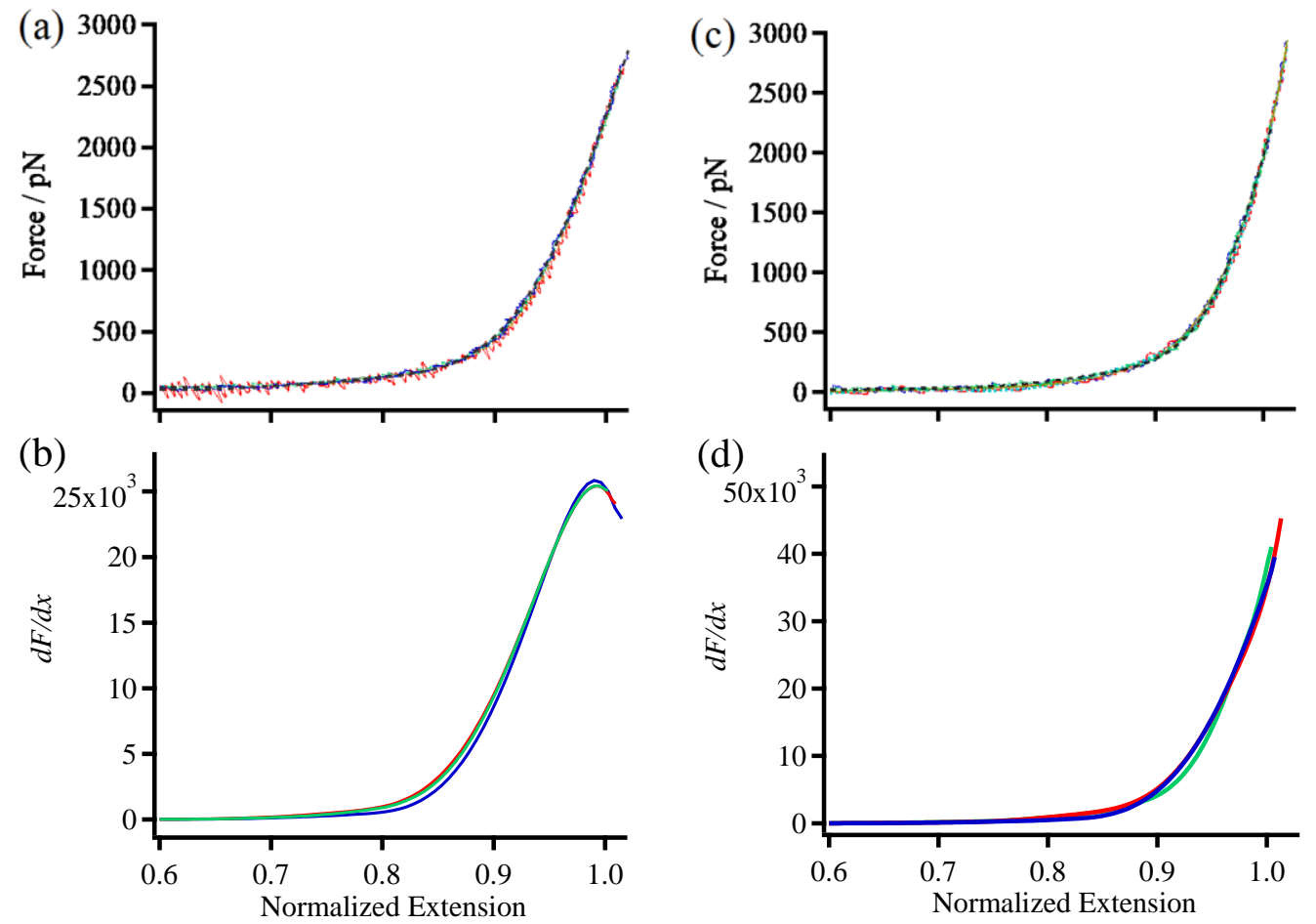

Figure S3. (a) Normalized single-chain $F-E$ curves of $\mathrm{NC}$ obtained in nonane. The dotted lines are the polynomial fitting curves of the experimental curves. (b) The $\mathrm{d} F / \mathrm{d} x$ of the polynomial fitting curves that shown in (a). (c) Normalized single-chain $F-E$ curves of NC obtained in DMSO. The dotted lines are the polynomial fitting curves of the experimental curves that shown in (c). (d) The $\mathrm{d} F / \mathrm{d} x$ of the polynomial fitting curves that shown in (c). 

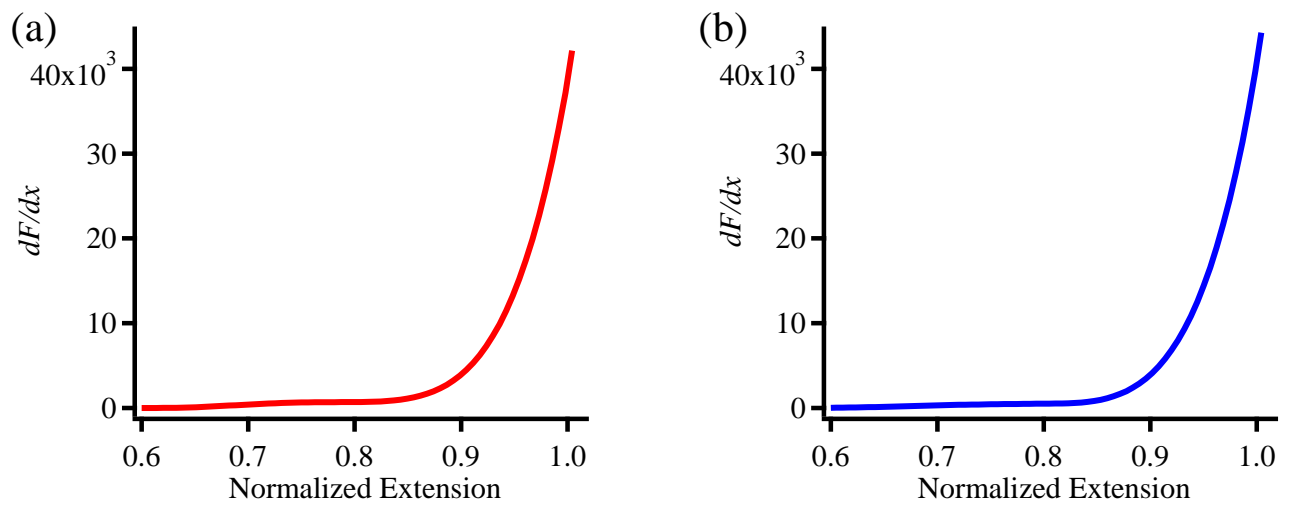

Figure S4. The $\mathrm{d} F / \mathrm{d} x$ of the polynomial fitting curves of the single-chain $F-E$ curves of CTA obtained in nonane (a) and DMSO (b). The experimental single-chain $F$-E curves of CTA in Figure $2 \mathrm{a}$ are firstly fitted by a polynomial to obtain the fitting curves.
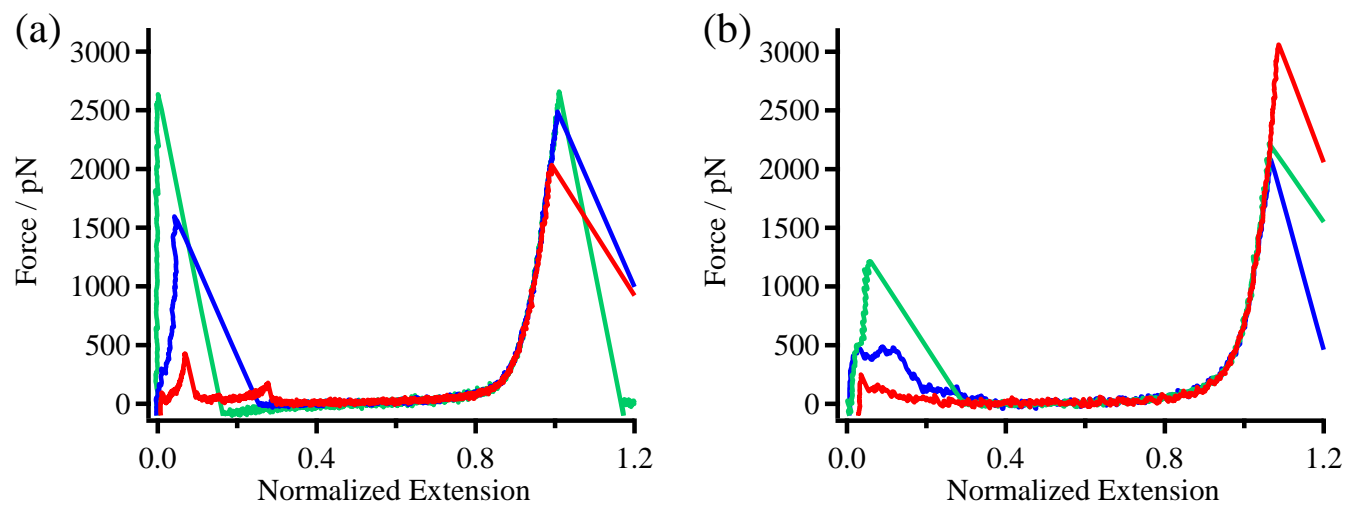

Figure S5. Normalized single-chain $F$ - $E$ curves of amylose obtained in nonane (a) and DMSO (b). 


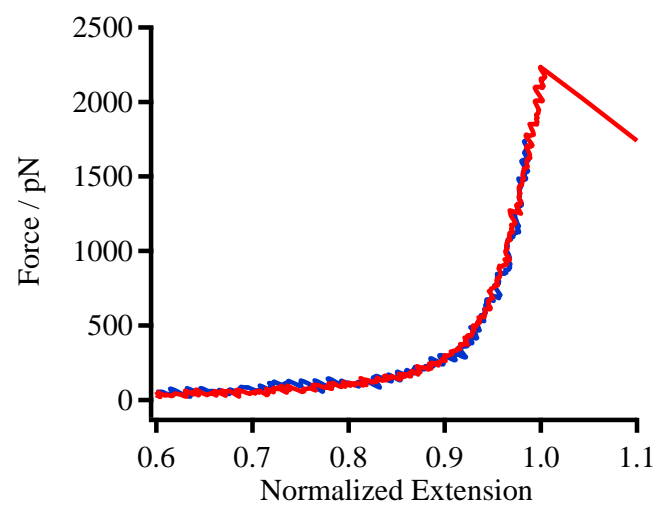

Figure S6. Normalized single-chain $F-E$ curves of ATA obtained in nonane (red) and DMSO (blue).
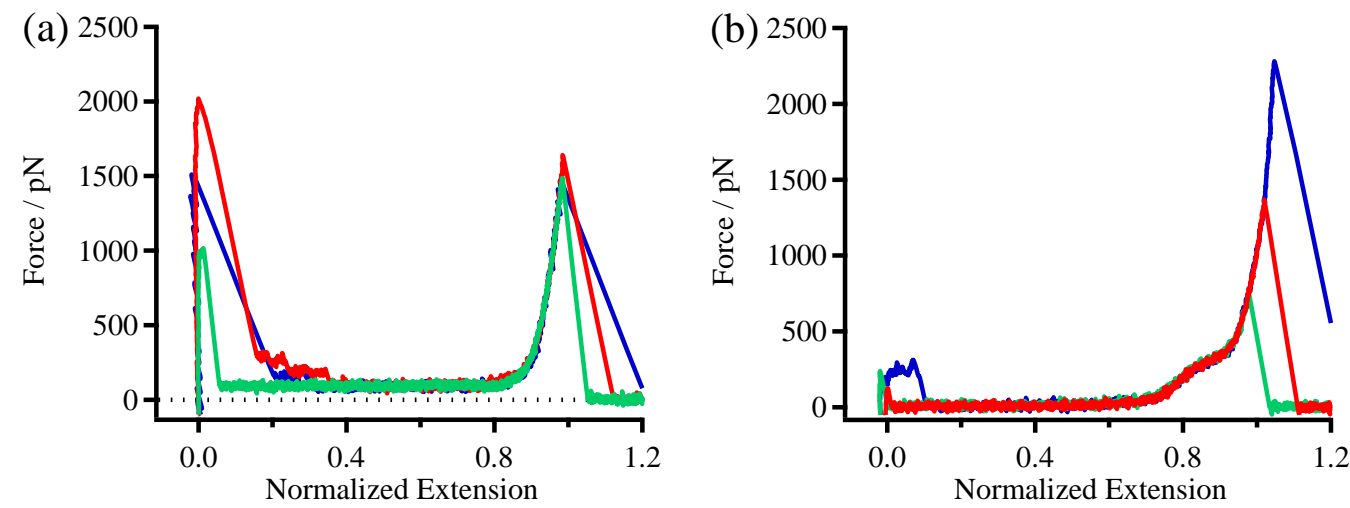

Figure S7. Normalized single-chain $F-E$ curves of NC (a) and amylose (b) obtained in DI water at $20{ }^{\circ} \mathrm{C}$. 


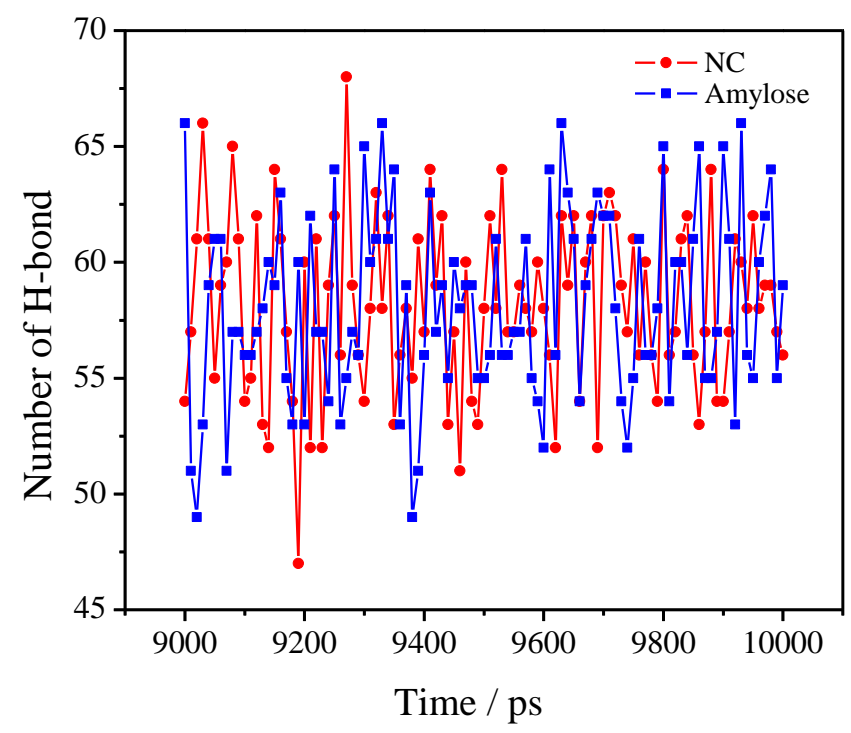

Figure S8. The number of H-bonds between polysaccharide and water. The time-averaged number of $\mathrm{H}$-bonds between water and 9-mer polysaccharide are 58.15 for NC and 58.07 for amylose, respectively.
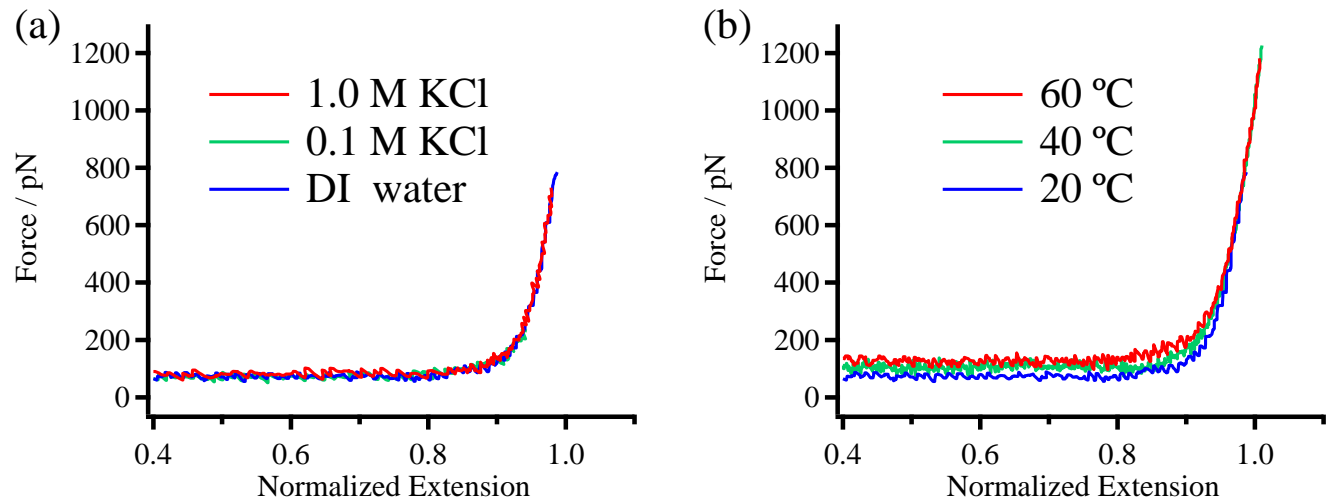

Figure S9. The $F-E$ curves of $\mathrm{NC}$ obtained in $\mathrm{KCl}$ solutions with different concentrations (a) and in DI water with different temperatures (b). The $F-E$ curves have been smoothed for clarity. 

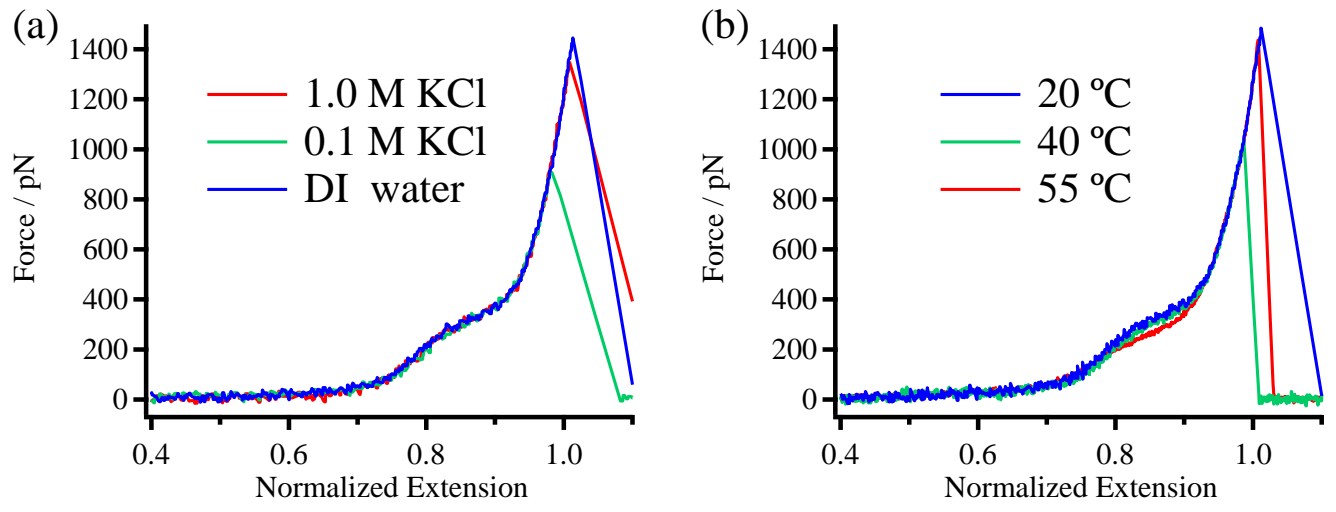

Figure S10. The $F-E$ curves of amylose obtained in $\mathrm{KCl}$ solutions with different concentrations (a) and in DI water with different temperatures (b). The $F$ - $E$ curves have been smoothed for clarity.

\section{REFERENCES}

(1) Imberty, A.; Chanzy, H.; Perez, S.; Buleon, A.; Tran, V., The Double-Helical Nature of the Crystalline Part of a-Starch. J. Mol. Biol. 1988, 201, 365-378.

(2) Winger, M.; Christen, M.; van Gunsteren, W. F., On the Conformational Properties of Amylose and Cellulose Oligomers in Solution. Int. J. Carbohydr. Chem. 2009, 307695.

(3) Luzar, A.; Chandler, D., Hydrogen-Bond Kinetics in Liquid Water. Nature 1996, 379, $55-57$.

(4) Li, I. T. S.; Walker, G. C., Interfacial Free Energy Governs Single Polystyrene Chain Collapse in Water and Aqueous Solutions. J. Am. Chem. Soc. 2010, 132, 6530-6540.

(5) Wu, S., Surface and Interfacial Tensions of Polymer Melts: I. Polyethylene, Polyisobutylene, and Polyvinyl Acetate. J. Colloid Interface Sci. 1969, 31, 153-161.

(6) Wu, S., Calculation of Interfacial Tension in Polymer Systems. J. Polym. Sci. Polym. 
Symp. 1971, 31, 19-30.

(7) Holthusen, D.; Haas, C.; Peth, S.; Horn, R., Are Standard Values the Best Choice? A Critical Statement on Rheological Soil Fluid Properties Viscosity and Surface Tension. Soil Tillage Res. 2012, 125, 61-71.

(8) Vanhazendonk, J. M.; Vanderputten, J. C.; Keurentjes, J. T. F.; Prins, A., A Simple Experimental-Method for the Measurement of the Surface-Tension of Cellulosic Fibers and Its Relation with Chemical-Composition. Colloid Surf. A-Physicochem. Eng. Asp. 1993, 81, 251-261.

(9) Reichardt, C., Solvatochromic Dyes as Solvent Polarity Indicators. Chem. Rev. 1994, 94 , 2319-2358. 